BULL. AUSTRAL. MATH. SOC.

\title{
Complemented hereditary radicals
}

\author{
Robert L. Snider
}

\begin{abstract}
The complemented elements of the lattice of hereditary radicals are characterized. A hypernilpotent complemented hereditary radical is the upper radical determined by a finite number of finite matrix rings. As a corollary, Stewart's characterization of radical semisimple classes is obtained. The methods are universal algebraic in nature.
\end{abstract}

In [14], we showed that the natural order on the class of all radicals for associative rings gives rise to a complete lattice in which the hereditary radicals form a complete sublattice, where for hereditary radicals $\alpha$ and $\beta$, the meet $(\alpha \wedge \beta)(R)=\alpha(R) \cap \beta(R)$ for any ring $R$. The semisimple class of the join $\alpha \vee \beta$ is the intersection of the semisimple class of $\alpha$ and the semisimple class of $\beta$. We also showed that the lattice of hereditary radicals is Brouwerian and hence distributive. In [14], we raised the question of characterizing the complemented elements of this lattice. In this paper, we completely characterize the complemented hereditary radicals by a detailed study of the polynomial identities of certain algebras. As an application, we quickly obtain a recent result of stewart [15] characterizing radical semisimple classes.

Our approach is somewhat universal algebraic in nature. We suggest the reader unfamiliar with this approach see Grätzer [8]. For elementary definitions and notions concerning radicals, see [7] or [11].

We shall always use lower case Greek letters to denote radicals. If $R$ is a ring, then the $n \times n$ matrix ring over $R$ will be denoted by $R_{n} \cdot|A|$ will denote the cardinality of the set $A$.

Received 16 November 1970. 
For a hereditary radical $\alpha$, let $\alpha^{*}$ denote its pseudocomplement where the pseudocomplement is the largest radical $\lambda$ such that $\alpha \wedge \lambda=0$. $\alpha^{*}$ must exist since our lattice is Brouwerian. If $\alpha$ has a complement, its complement must necessarily be $\alpha^{*}$. Suppose now that $\alpha$ is complemented. Since $\left(\alpha \vee \alpha^{*}\right)\left(z_{0}\right)=z_{0}$ and $\left(\alpha \wedge \alpha^{*}\right)\left(z_{0}\right)=0$ where $z_{0}$ is the integers with zero multiplication, we have either $\alpha\left(z_{0}\right)=z$ and $\alpha^{*}\left(z_{0}\right)=0$ or $\alpha\left(z_{0}\right)=0$ and $\alpha^{*}\left(z_{0}\right)=z_{0}$. In the remainder of the paper, we shall always assume $\alpha\left(z_{0}\right)=z_{0}$. This means that $\alpha$ is hypernilpotent. We shall characterize the hypernilpotent complemented radicals. A non-hypernilpotent complemented hereditary radical is then just the complement of a hypernilpotent one.

Let $\alpha$ be a hereditary radical. In [4], Andrunakievic constructed the largest radical $\alpha^{\prime}$ among all those radicals $\beta$ with $\alpha(R) \cap \beta(R)=0$ for every ring $R$. Clearly if $\alpha^{\prime}$ is hereditary, $\alpha^{\prime}=\alpha^{*}$. If $\alpha$ is hypernilpotent, he showed that $\alpha^{\prime}$ and $\alpha^{\prime \prime}$ were hereditary. Therefore, if $\alpha$ is also complemented, we have $\alpha^{\prime}=\alpha^{*}$ and $\alpha^{\prime \prime}=\alpha^{* *}=\alpha$. It then follows from [4] that

$$
\alpha^{*}(R)=\Pi\{I: R / I \text { is subdirectly irreducible with a-radical heart }\} \text {, }
$$

and

$$
\begin{gathered}
\alpha(R)=\alpha^{* *}(R)=\Pi\{I: R / I \text { is subdirectly irreducible with } \\
\alpha \text {-semisimple heart }\} .
\end{gathered}
$$

THEOREM 1. If $S$ is an a-semisimple simple ring where $\alpha$ is a complemented hypernilpotent radical, then $S$ is finite.

Proof. Let $C$ be the centroid of $S, C$ is a field since $S$ is simple and $S$ can be regarded as an algebra over $C$. In [14], we showed that $C$ is finite. We show $S$ satisfies a polynomial identity over $C$. Suppose not. Let $F$ be the free algebra over $C$ with $\max \left\{\kappa_{0},|S|\right\}$ generators. Let $f \neq 0$ be in $F$; then there exists a homomorphism $h: F \rightarrow S$ such that $h(f) \neq 0$ since $S$ does not satisfy a polynomial identity. If $x_{1}, \ldots, x_{n}$ are the generators of $F$ in the expression of $f$, we define $h^{\prime}: F+S$ by $h^{\prime}\left(x_{i}\right)=h\left(x_{i}\right), i=1, \ldots, n$ and defining $h^{\prime}$ on the other generators making $h^{\prime}$ onto. This can be done 
since $F$ has at least as many generators as $S$ has elements. Hence $n\{$ kerh $: h: F \rightarrow S$ is onto $\}=0$, that is $F$ is a subdirect sum of copies of $S$. Therefore $\alpha(F)=0$. Let $H$ be an infinite field containing $C$ and $G$ a simple ring which satisfies no polynomial identities but contains $H$ in its center (for example, a division ring over $H$ infinite dimensional over its center). Let $F^{\prime}$ be the free ring with $\max \left\{s_{0},|S|,|G|\right\}$ elements. Repeating the above argument, we obtain $\alpha\left(F^{\prime}\right)=0$. Also $F^{\prime}$ is a subdirect sum of copies of $G$. $\alpha(G)=G$ since $G$ has infinite centroid [14]. Therefore $\alpha^{*}(G)=0$ and hence $\alpha^{*}\left(F^{\prime}\right)=0$. We then have $\left(\alpha \vee \alpha^{*}\right)\left(F^{\prime}\right)=0$, contradicting the fact that $\alpha \vee \alpha^{*}=1$. (1 is the radical for which all rings are radical.) Since $S$ satisfies a polynomial identity, $S$ is primitive by a theorem of Herstein in [13]. By a theorem of Kaplansky [9], $S$ is finite dimensional over its center which must be $C$. Therefore $S$ is finite since $C$ is finite.

LEMMA 2. GF $\left(p^{q}\right)_{n}$ satisfies the identity $x^{n+r}-x^{n}=0$ where $r$ is the exponent of $\mathrm{GL}\left(n, p^{q}\right)$.

Proof. Let $\mathrm{GF}\left(p^{q}\right)_{n}$ act on an $n$ dimensional $\mathrm{GF}\left(p^{q}\right)$ vector space $V$. If $A$ is in $\mathrm{GF}\left(p^{q}\right)_{n}$, then we have a descending chain of subspaces, $A V \supseteq A^{2} V \supseteq \ldots \supseteq A^{n_{V}} \supseteq \ldots$ which must terminate at $A^{n} V$ since $V$ has dimension $n$. A then induces an automorphism $\phi$ on $A^{n} V$ which can be extended to an automorphism $\bar{\phi}$ of $V$. Hence $\bar{\phi}^{r}=1$. For any $v$ in $V$, we have $A^{n}(v)=\phi^{r} A^{n}(v)=\vec{\phi}^{r} A^{n}(v)=A^{n+r}(v)$. Therefore $A^{n}=A^{n+r}$.

LEMMA 3. Let $V$ be the variety of $\mathrm{GF}(p)$ algebras generated by $\mathrm{GF}\left(p^{q}\right)_{n}$. If $S$ is in $V$ and $A$ is a subalgebra of $S$ isomorphic to $\mathrm{GF}(p)_{n}$, then $A$ annihilates every nilpotent ideal of $S$.

Proof. The proof is by induction on the index of nilpotence. Let $N$ be an ideal of $S$ and suppose $N^{2}=0$. Let $e$ denote the identity of $A$. It is sufficient to show that $e N=N e=0$. First suppose elle $\neq 0$. Since the action of $\mathrm{GF}(p) e$ is the same on both sides of eNe, 
we can regard eNe as a left $A \otimes_{G F(p)} A^{\circ}$ module where $A^{\circ}$ denotes the opposite algebra of $A$. Multiplication is by $(a \otimes b)(e n e)=a e n e b$. $A \otimes_{\mathrm{GF}(p)} A^{0} \cong \mathrm{GF}(p)_{n^{2}}$. All modules over $\mathrm{GF}(p)_{n^{2}}$ are the sum of simple modules; hence elle contains a simple submodule. All simple modules over $G F(p)_{n^{2}}$ are isomorphic; hence we may identify one such simple submodule with all

$$
\left[\begin{array}{l}
x_{I} \\
\cdot \\
x_{n}
\end{array}\right] \otimes_{\mathrm{GF}(p)}\left[\begin{array}{l}
y_{I} \\
\cdot \\
y_{n}
\end{array}\right] .
$$

Identify $A$ with $G F(p)_{n}$. Let $I_{s}$ denote the $s \times s$ identity matrix. Let

$$
y=\left[\begin{array}{ll}
0 & 0 \\
I_{n-1} & 0
\end{array}\right]+\left[\begin{array}{l}
1 \\
1 \\
\cdot \\
\cdot \\
1
\end{array}\right] \otimes\left[\begin{array}{l}
1 \\
0 \\
\cdot \\
\cdot \\
0
\end{array}\right]
$$

which is in $S$. Recalling $N^{2}=0$, we compute

$$
\begin{gathered}
y^{2}=\left[\begin{array}{ll}
0 & 0 \\
I_{n-2} & 0
\end{array}\right]+\left[\begin{array}{l}
0 \\
1 \\
\cdot \\
\cdot \\
\cdot \\
1
\end{array}\right] \otimes\left[\begin{array}{l}
1 \\
0 \\
\cdot \\
\cdot \\
0
\end{array}\right], \\
y^{n-1}=\left[\begin{array}{ll}
0 & 0 \\
\\
1 & 0
\end{array}\right]+\left[\begin{array}{l}
0 \\
\cdot \\
\cdot \\
\dot{1} \\
1
\end{array}\right] \otimes\left[\begin{array}{l}
1 \\
0 \\
\cdot \\
\cdot \\
\cdot \\
0
\end{array}\right],
\end{gathered}
$$




$$
y^{n}=0+\left[\begin{array}{l}
0 \\
\cdot \\
\cdot \\
\cdot \\
0 \\
1
\end{array}\right] \otimes\left[\begin{array}{l}
1 \\
0 \\
\cdot \\
\cdot \\
\cdot \\
0
\end{array}\right]
$$

and $y^{n+1}=0$.

By the previous Iemma, we have

$$
0 \neq\left[\begin{array}{l}
0 \\
\cdot \\
\cdot \\
0 \\
1
\end{array}\right] \otimes\left[\begin{array}{l}
1 \\
0 \\
\cdot \\
\cdot \\
0
\end{array}\right]=y^{n}=y^{n+r}=0,
$$

a contradiction.

Therefore $e N e=0$. Suppose now that $e N \neq 0$. eN is a unital $A$ module and hence has a simple submodule which we may identify with all $\left[\begin{array}{l}x_{1} \\ \cdot \\ \cdot \\ x_{n}\end{array}\right]$ since all simple $A$ modules are isomorphic. Let

$$
z=\left[\begin{array}{ll}
0 & 0 \\
& \\
I_{n-1} & 0
\end{array}\right]+\left[\begin{array}{l}
I \\
\cdot \\
i \\
I
\end{array}\right]
$$

As before using elle $=0$, we compute

$$
z^{n}=\left[\begin{array}{c}
0 \\
\cdot \\
\cdot \\
\dot{1}
\end{array}\right]
$$

and $z^{n+1}=0$. We now obtain

$$
0 \neq\left[\begin{array}{c}
0 \\
\cdot \\
\cdot \\
i
\end{array}\right]=z^{n}=z^{n+r}=0
$$


as before. Therefore $e N=0$. Similarly $N e=0$.

Suppose now that $A$ annihilates all nilpotent ideals of index less than $k$. Suppose $N$ has index $k$. Clearly $A \cap N=0$. Passing to $S / N^{2}$, we have $A$ isomorphically embedded in $S / N^{2}$. Therefore by the above, we have $\left(e+N^{2}\right) N / N^{2}=0$ or $e N \subseteq N^{2} . N^{2}$ has index of nilpotence less than $k$; therefore, our induction hypothesis applies. We obtain $e N=e^{2} N \subseteq e N^{2}=0$. Similarly $N e=0$.

LEMMA 4. Let $F$ be a finite field of characteristic $p$. If $U$ is the variety of $\mathrm{GF}(p)$ algebras generated by $F_{n}$, then the free algebra over $V$ with $\aleph_{0}$ generators can be described as follows: let $A_{k}=\left[x_{i j}^{(k)}\right]$ be the $n \times n$ matrix with entries commuting indeterminates with $\left(x_{i j}^{(n)}\right)|F|=x_{i j}^{(k)}$ and $p x_{i j}^{(k)}=0$. The free algebra is the algebra generated by the $A_{k}$ with ordinary matrix multiplication and addition.

REMARK. Amitsur [2] states this without proof for infinite fields of characteristic not necessarily $p$.

Proof. Let $R$ be the algebra generated by the $A_{k}$ 's.$R$ is clearly free over $V$ since every substitution by elements of $F$ for the

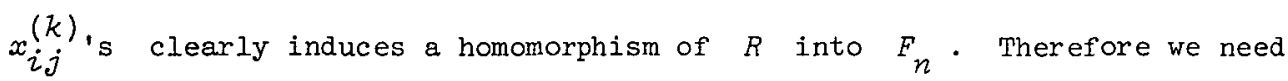
only show $R$ is in $V$. To see this, consider the algebra $T$ generated by the $x_{i j}^{(k)}$ !s. $T$ is clearly the free algebra of $U(F)$. Hence $\operatorname{Id}(F)=\operatorname{Id}(T)$. From [12, Theorem 3], $\operatorname{Id}\left(E_{n}\right)=\operatorname{Id}\left(T_{n}\right) \cdot$ Clearly $R \subseteq T_{n}$. Therefore $R$ is in $V$ since $T_{n}$ is.

For a class of rings $H$, we denote the upper radical of $M$ by $M M$.

THEOREM 5. Let $F=G F\left(p^{q}\right)$. If $\alpha=U\left\{F_{n}\right\}$, then $\alpha$ is $\alpha$ complemented hereditary radical.

Proof. $\alpha \wedge \alpha^{*}=0$. Suppose then that $\alpha \vee \alpha^{*}<1$. Since the semisimple class of the join is the intersection of the semisimple classes [14], there exists a ring $R \neq 0$ such that $\alpha(R)=\alpha^{*}(R)=0 .\left\{F_{n}\right\}$ is 
a special class since every class of simple rings with unity is special [4], hence $\alpha(R)=\cap\left\{I: R / I \cong F_{n}\right\}$; that is, $R$ is a subdirect sum of copies of $F_{n}$. It follows that $R$ is in the variety generated by $F_{n}$. Also since $\alpha$ is hypernilpotent, we have $\alpha^{*}=\alpha^{\prime}$ where $\alpha^{\prime}$ is the complementary radical of Andrunakievič [4]. Therefore $\alpha^{*}(R)=\Pi\{I: R / I$ is subdirectly irreducible with $\alpha$-radical heart $\}$.

By Lemma 2, $F_{n}$ and hence $R$ satisfies the identity $x^{n+r}-x^{n}=0$ where $r$ is the exponent of $\operatorname{GL}(n, F)$. Therefore $R$ is a P.I. algebra and also an algebraic algebra and hence $R$ is locally finite [10]. There exists an epimorphism $h: R \rightarrow F_{n}$. Let $\left\{x_{i}\right\}$ be a complete set of liftings of $F_{n}$. The subalgebra $\left\langle x_{i}\right\rangle$ generated by $\left\{x_{i}\right\}$ is finite since $R$ is locally finite. $h \mid\left\langle x_{i}\right\rangle$ is onto. Let $J\left(\left\langle x_{i}\right\rangle\right)$ denote the Jacobson radical of $\left\langle x_{i}\right\rangle . R / J\left(\left\langle x_{i}\right\rangle\right)$ is a separable algebra; hence by a generalization of the Principal Theorem of Wedderburn [1], $\left\langle x_{i}\right\rangle$ and hence $R$ contains a subalgebra $A$ isomorphic to $F_{n}$.

$R$ is a subdirect sum of subdirectly irreducible rings $\left\{S_{j}\right\}$ with $\alpha$-radical hearts. The image of $A$ must be nonzero in some $S_{i} \cdot A$ is simple; hence the image is an isomorphic copy. We suppose now that $A \subseteq S_{i}$. Let $H$ be the heart of $S_{i}$. Suppose $H^{2}=H . H$ is then a simple ring. $H$ satisfies a polynomial identity since $R$ does. By a theorem of Herstein in [13], $H$ is primitive and hence by a theorem of Kaplansky [9], $H$ is isomorphic to $D_{m}$ where $D$ is a division ring finite dimensional over its center $C \cdot C$ must satisfy $x^{n+r}-x^{n}=0$. Therefore $C$ is finite and $C=D . C_{m}$ has a unit. It follows that $C_{m}$ is a direct summand of $S \cdot S_{i}$ is subdirectly irreducible, hence $C_{m}=S_{i}$. Recall $A \subseteq C_{m}$. Since $A$ satisfies no identities of degree less than $2 n$ [3], we have $n \leq m$ since $C_{m}$ satisfies the standard identity of degree $2 m$ [3]. Also $C_{m}$ satisfies all identities of $F_{n}$ 
since $R$ does. Therefore $m \leq n$. Since $A \subseteq C_{n}, V(A) \subseteq V\left(C_{n}\right)$ and clearly by construction $V\left(C_{n}\right) \subseteq V(A)=V\left(F_{n}\right)$. Let $\left\langle A_{n}\right\rangle$ be the free algebra with $\aleph_{0}$ generators of $V\left(F_{n}\right)$ described in the previous lemma and $\left\langle B_{k}\right\rangle$. the corresponding free algebra for $V\left(C_{n}\right)$. The mapping $A_{k} \rightarrow B_{k}$ induces an isomorphism. It is clear then that $C=F$. This is impossible since $C_{n}=S_{i}$ was assumed to have $\alpha$-radical heart, but $\alpha\left(F_{n}\right)=0$. Therefore $H^{2}=0$.

Let $e$ denote the identity of $A$. Let $X$ be the collection of all idempotents $x$ of $e S_{i} e$ such that $\alpha x=x a$ for all $a$ in $A . X$ is not empty since $e$ is in $X$. Let $I=\{\{A x: x$ is in $X\}$. The Jacobson radical $J\left(S_{i}\right)$ of $S_{i}$ is nilpotent since $S_{i}$ is an algebraic algebra of bounded index [4]. e annihilates $J\left(S_{i}\right)$ by Lemma 3 . It follows that $e S_{i} e \cap J\left(S_{i}\right)=0$. Clearly $I \subseteq e S_{i} e$. It is then sufficient to show that $I$ is an ideal of $S_{i}$ since this will contradict the subdirect irreducibility of $S_{i}$. Let $x$ be in $X$ and $y$ in $S_{i}$. Note that $A x=x A$; hence it sufficies to show $A y \subseteq I$. Consider the subalgebra $\langle A, y\rangle$ generated by $A$ and $y \cdot\langle A, y\rangle$ is finite dimensional since $S_{i}$ is an algebraic algebra which satisfies a polynomial identity and hence is locally finite $[10] .\langle A, y\rangle / J(\langle A, y\rangle)$ is separable where $J(\langle A, y\rangle)$ is the Jacobson radical of $\langle A, y\rangle$. By the Principal Theorem of Wedderburn, $\langle A, y\rangle$ contains a subalgebra $B$ which is isomorphic to $\langle A, y\rangle / J(\langle A, y\rangle)$ and $\langle A, y\rangle=B+J(\langle A, y\rangle)$. The previous sum is a vector space direct sum. By Lemma $3, A$ annihilates the nilpotent ideal $J(\langle A, y\rangle)$ since $A \supseteq \mathrm{GF}(p)_{n} \cdot e=z+j$ with $z$ in $B$ and $j$ in $J(\langle A, y\rangle)$. The projection of $A$ into $B$ is a ring homomorphism since $J(\langle A, y\rangle)$ is an ideal. Therefore $\operatorname{Im}(A)$ is isomorphic to $A$ and hence $z j=j z=0$ whenever $a=z+k$ for some $a$ in $A$. We then have $e=e^{2}=e(z+j)=e z=(z+j) z=z^{2}=z$ for $e=z+j$. Hence $A \subseteq B$. By the Wedderburn-Artin Theorem $B=C_{1} \oplus C_{2} \oplus \ldots \oplus C_{r}$ where $C_{i}$ are matrix rings over division rings. 
The projection of $A$ on each factor is either 0 or an embedaing. As before, if $F_{n}=A \subseteq C_{i}$, we have $A=C_{i}$. By renumbering if necessary, we have $B=C_{1} \oplus C_{2} \oplus \ldots \oplus C_{t} \oplus C_{t+1} \oplus \ldots \oplus C_{r}$ where $F_{n} \cong C_{i}$ and $e c=c e, i \leq t$, and $e c_{j}=0, j>t$. Then $e c_{i} e=c$ and $c_{i} a=a c_{i}$ for each $a$ in $A$ where $c_{i}$ is the identity of $c_{i}, i \leq t$. Hence $c_{i}$ is in $x$ and $A c_{i}=c_{i}$. Now $y=b+j \cdot a y=a b+a j=a b$ is in $\left(c_{1} \oplus c_{2} \oplus \ldots \oplus C_{t}\right) b \subseteq C_{1} \oplus C_{2} \oplus \ldots \oplus C_{t} \subseteq I$. Therefore $I$ is an ideal.

THEOREM 6. Let $\alpha$ be a hypernilpotent radical. $\alpha$ is complemented if and only if $\alpha$ is the upper radical determined by a finite number of matrix rings over finite fields.

Proof. First suppose $\alpha=u\left\{F_{n_{i}}^{(i)}\right\}_{i=1}^{r}$. Let $\alpha_{i}=u\left\{F_{n_{i}}(i)\right\} \cdot \alpha_{i}$ is complemented by the previous theorem. $\alpha=\alpha_{1} \wedge \alpha_{2} \wedge \ldots \wedge \alpha_{r}$ [14]. Clearly $\beta=\alpha_{1}^{*} \vee \alpha_{2}^{*} \vee \ldots \vee \alpha_{r}^{*}$ is the complement of $\alpha$.

Conversely suppose $\alpha$ has a complement. $\alpha=\alpha^{\prime \prime}$ since $\alpha$ is hypernilpotent. It follows that $\alpha$ is the upper radical determined by the subdirectly irreducible rings with $\alpha^{*}$-radical hearts. Let $S$ be such a ring with heart $H$. Since $\alpha$ is hypernilpotent, $S$ is a semiprime ring and hence $H^{2}=H$. Therefore $H$ is a simple ring. Therefore by Theorem $1, H$ is finite and hence is a matrix ring over a finite field. $H$ has an identity and hence $H$ is a direct summand of $S$. Therefore $H=S$. We now have that $\alpha$ is the upper radical determined by matrix rings over finite fields. We must show that their number is finite.

Suppose first of all that for some prime $p$, the number of a-semisimple simple rings is finite. Let $\left\{\begin{array}{c}(i) \\ n_{i}\end{array}\right\}$ be these rings. We first show $n$ is bounded. Suppose not. It follows that no identity over $G F(p)$ can be satisfied by all the $F_{n_{i}}^{(i)}$ since all identities satisfied by $F_{n_{i}}^{(i)}$ have degree at least $2 n_{i}[3]$. Consider the free $G F(p)$ 
algebra $G$ with $c$ (cardinality of the continuum) generators. As in the proof of Theorem l, we see that $G$ is a subdirect sum of the $F_{n_{i}}^{(i)}$. Therefore $\alpha(G)=0$. If on the other hand $S$ is a simple algebra satisfying no polynomial identities over $G F(p)$ and $|S| \leq c$ (for example, all linear transformations of finite rank on a vector space of countably infinite dimension over $\mathrm{GF}(p))$, then $\alpha(S)=S \cdot \alpha$ is complemented; hence $\alpha^{*}(S)=0$. As before we see that $G$ is a subdirect sum of copies of $S$. Therefore $\alpha(G)=\alpha^{*}(G)=\left(\alpha \vee \alpha^{*}\right)(G)=0$, a contradiction since $\alpha \vee \alpha^{*}=1$. Therefore $n_{i}$ is bounded. Hence for some $n$, there is an infinite number of fields $F^{(j)}$ of characteristic $p$ with $n_{j}=n$. We distinguish two cases.

Case I. All but finitely many finite fields of characteristic $p$ are in $\left\{F^{(j)}: n_{j}=n\right\}$.

Case II. Infinitely many finite fields of characteristic $p$ are not $E^{(j)}$ is.

Case I. Let $C$ be the direct limit of the ${ }_{F}^{(j)}$ 's where the $F^{(j)}$ 's form a direct system with the inclusion maps. $C$ exists since all but finitely many finite fields of characteristic $p$ are represented. $C$ is a field and $C_{n}$ is the direct limit of the ${ }_{F}^{(j)}$. Clearly $F_{n}^{(j)} \subseteq C_{n}$, hence $\operatorname{Id}\left(C_{n}\right) \subseteq \operatorname{MId}\left(F_{n}^{(j)}\right)$. Also if $p\left(x_{1}, \ldots, x_{n}\right)=0$ is an identity for each $F_{n}^{(j)}$ then since $c_{n}$ is a direct limit of the $F_{n}^{(j)}$, we have $p\left(x_{1}, \ldots, x_{n}\right)$ is an identity for $c_{n}$. Hence $v\left(C_{n}\right)=v\left\{F_{n}^{(j)}\right\}$. Therefore the free $\mathrm{GF}(p)$ algebra $H$ over this variety with $\aleph_{0}$ generators is a subdirect sum of the $F_{n}^{(j)}$ 's as before. Therefore $\alpha(H)=0$. Now $\alpha^{*}\left(C_{n}\right)=0$ and $H$ is a subdirect sum of copies of $C_{n}$, hence $\alpha^{*}(H)=0=\alpha(H)=\left(\alpha \vee \alpha^{*}\right)(H)$, a contradiction. 
Case II. Consider the polynomial ring $(\mathrm{GF}(p)[x])_{n} \cdot(\mathrm{GF}(p)[x])_{n}$ is a subdirect sum of the $F_{n}^{(j)}$ and hence we have $\alpha(G F(p)[x])_{n}=0$. Also since there are infinitely many $F_{n}$ with $\alpha^{*}\left(F_{n}\right)=0$, we have $\alpha^{*}(G F(p)[x])_{n}=0$. Again we have $\left(\alpha \vee \alpha^{*}\right)(G F(p)[x])_{n}=0$, a contradiction.

We now have that for each prime $p$, there are only finitely many o-semisimple simple rings of characteristic $p$. We now show that only finitely many $p$ are represented. Suppose not. Let $K_{n_{i}}^{(i)}$ be the a-semisimple simple rings. We first show $n_{i}$ is bounded above. Suppose not. We show that no polynomial identity over the integers is satisfied by all the $k_{n_{i}}^{(i)}$. Consider $g\left(x_{1}, \ldots, x_{n}\right), g\left(x_{1}, \ldots, x_{n}\right)=0$ $(\bmod p)$ for only finitely many primes $p$. Let $s$ be the degree of $g\left(x_{1}, \ldots, x_{n}\right)$. We can find some prime $p$ such that $g\left(x_{1}, \ldots, x_{n}\right) \neq 0$ $(\bmod p)$ and there is a $K_{n_{i}}^{(i)}$ of characteristic $p$ with $2 n_{i} \geq s$. Since $K_{n_{i}}^{(i)}$ satisfies no identity of degree less than $2 n_{i}$, $g\left(x_{1}, \ldots, x_{n}\right)$ is not identity in $k_{n_{i}}$. As before the free algebra over the integers with sufficiently many generators is a subdirect sum of the $K_{n_{i}}^{(i)}$ and the subdirect sum of copies of some infinite simple ring which satisfies no identities over the integers. It follows that the free algebra is $\alpha \vee \alpha^{*}$-semisimple. Again this is a contradiction and hence $n_{i}$ is bounded. As before, we must have infinitely many $n_{i}$ equal $n$ for some positive integer $n$. Again we have the polynomial ring $(z[x])_{n}$ ( $z$ denotes the integers) is a subdirect sum of the $K_{n_{i}}^{(i)}$ 's . Also for each $p$, we can pick $G_{n}^{(p)}$ with $\alpha\left(G_{n}^{(p)}\right)=G_{n}^{(p)}$ where $G$ is a finite field of characteristic $p .(Z[x])_{n}$ is also a subdirect sum of the 
$G_{n}^{(j)}$. Therefore $\alpha\left(z[x]_{n}\right)=\alpha^{*}\left(z[x]_{n}\right)=0$, a contradiction.

We are now able to obtain a recent theorem of Stewart [15] characterizing radical semisimple classes. A radical semisimple class $M$ is a radical class which is simultaneously a semisimple class for some other radical.

THEOREM 7 (Stewart). $M$ is a radical semisimple class if and only if there exists an integer $n$ such that

$$
M=\left\{R: x^{n}=x \text { for every } x \text { in } R\right\} \text {. }
$$

Proof. It is easy to verify that if $M$ is as above, then $M$ is a radical semisimple class. Suppose then that we are given a radical semisimple class $M . M$ is closed under homomorphic images and subdirect sum. Therefore $M$ is a variety. Let $\beta$ be the radical with $M$ as its radical class and $\alpha$ the radical whose semisimple class is $M$. We show that $I^{2}=I$ for every ring $I$ of $M$. Suppose not. We then have $I / I^{2}$ is in in and $I / I^{2}$ is a zero ring. Every ideal of $Z_{0}$, the integers with zero multiplication, can be mapped into $I / I^{2}$ with nonzero image. Since $M$ is a semisimple class we have $Z_{0}$ is in $M$. This implies $B$ is larger than the Baer lower radical. Armendariz has shown [6] that this implies that $M$ is all rings, a contradiction. $I^{2}=I$ implies $\beta$ is subidempotent [4], hence the complementary radical $\beta^{\prime}$ is hereditary [4] and hence $\beta^{\prime}=\beta^{*}$. Clearly $\beta^{\prime} \geq \alpha$. It is then clear that $\beta^{*} \vee \beta=1$ and $\beta$ is complemented. We then have that $\beta^{*}$ is the upper radical determined by a finite number of finite matrix rings $\left\{F_{n_{i}}(i)\right\}_{i=1}^{n}$. Each $F_{n_{i}}^{(i)}$ is in $M$ since $B\left(F_{n_{i}}^{(i)}\right)=F_{n_{i}}^{(i)}$ and hence all subrings are in $M$ since $M$ is a variety. If any $n_{i}>1$, then $M$ must contain rings with zero multiplication, but $I^{2}=I$ for every ring in $M$, a contradiction. Therefore $n_{i}=1$. Let $n$ be the least common multiple of the $\left|F^{(i)}\right|$ 's $n$ clearly is the $n$ demanded in the theorem. 


\section{References}

[1] A. Adrian Albert, Structure of algebras (Colloquium Publ. 24, Amer. Math. Soc., New York, 1939).

[2] S.A. Amitsur, "Associative rings with identities", Some aspects of ring theory, ed. I.N. Herstein, (Centro Internazionalo Matematico Estivo, Roma, 1966).

[3] A.S. Amitsur and J. Levitzki, "Minimal identities for algebras", Proc. Amer. Math. Soc. 1 (1950), 449-463.

[4] V.A. Andrunakievič, "Radicals of associative rings. I", (Russian), Mat. Sb. N.S. 44 (86) (1958), 179-212.

[5] Richard F. Arens and Irving Kaplansky, "Topological representation of algebras", Trans. Amer. Math. Soc. 63 (1948), 457-481.

[6] E.P. Armendariz, "Closure properties in radical theory", Pacific $J$. Math. 26 (1968), 1-7.

[7] Nathan Divinsky, Rings and radicals (University of Toronto Press, Toronto, 1965).

[8] George Grätzer, Universal algebra (Van Nostrand, Princeton, New Jersey; Toronto; London; Melbourne; 1968).

[9] Irving Kaplansky, "Rings with a polynomial identity", Buzl. Amer. Math. Soc. 54 (1948), 575-580.

[10] Irving Kaplansky, Topological representation of algebras. II", Trans. Amer. Math. Soc. 68 (1950), 62-75.

[11] A.G. Kurós, "Radicals of rings and algebras", (Russian), Mat. Sb. N.S. 33 (75) (1953), 13-26.

[12] U. Leron and A. Vapne, "Polynomial identities of related rings", Israel J. Math. 8 (1970), 127-137.

[13] M. Susan Montgomery, "The Lie structure of simple rings with involution of characteristic 2", (Ph.D. thesis, University of Chicago, Chicago, 1969).

[14] Robert L. Snider, "Lattices of radicals", (to appear). 
[15] Patrick N. Stewart, "Semi-simple radical classes", Pacific J. Math. 32 (1970), 249-254.

University of Miami,

Coral Gables,

Florida, USA. 